\title{
A Methodology for assessing Video transmission Energy Consumption and Quality
}

\author{
Vitor Bernardo \\ Center for Informatics and Systems \\ University of Coimbra \\ Polo II, Pinhal de Marrocos, \\ 3030-290 Coimbra, Portugal \\ E-mail:vmbern@dei.uc.pt
}

\author{
Marilia Curado \\ Center for Informatics and Systems \\ University of Coimbra \\ Polo II, Pinhal de Marrocos, \\ 3030-290 Coimbra, Portugal \\ Email: marilia@dei.uc.pt
}

\begin{abstract}
Video traffic has been growing significantly and it is expected to be a large share of the future Internet traffic, within an increasingly mobile environment with scarce resources. In this context, new challenges arise, and energy-efficiency is becoming a key factor for the successful deployment of mobile networks. The development and validation of mechanisms for energyefficient video transmission require the measurement of the energy consumption needed for video transmission, considering also the end-user Quality of Experience. This issue is of utmost importance, as users will tailor their application and network usage behaviors based on the perceived quality of the services and on the capabilities of their devices. In this work, an integrated empirical methodology to assess the video transmission energy consumption and quality is proposed. The developed methodology was validated on two distinct testbeds, one with IEEE 802.11 and the other with IEEE 802.16e. Results showed that the proposed methodology enables an accurate assessment of energy consumption with different technologies, while measuring the end-user Quality of Experience. Another contribution of the proposed methodology is the capability to gather data to support the creation and validation of realistic simulation models.

Index Terms-Energy, Measurement, Methodology, Video, Quality of Experience, Testbed, Wireless, WiMAX
\end{abstract}

\section{INTRODUCTION}

The widespread deployment of new multimedia systems, applications such as Internet Protocol Television (IPTV), Video Conferencing, Video on Demand (VoD) or Voice over IP (VoIP) are replacing very quickly the legacy systems. The video transmission over the Internet is expected to represent around $66 \%$ of the overall Internet traffic by 2014 [1].

Together with the increasing video traffic, the future Internet also encompasses several requirements related with energyefficiency. Energy consumption raises issues to the operator related with operating expenses and to the end-user, which aims to run its device without recharging for as long as possible [2]. Many energy-efficiency techniques for wireless networks can be found in the literature, which include lowerlayers adaptation and optimization, as well as, end-users applications specifically designed to save energy, for instance, by performing smart sleep.

Additionally, it is important for both operator and end-user to measure the video applications quality, in order to assess the capabilities of a certain network to provide the needed (or contracted) video quality, while minimizing the energy consumption of the whole system.

This work proposes an empirical methodology for assessing the energy consumption and video quality. The proposed methodology aims to contribute for the development of techniques to minimize the energy consumption, keeping the enduser Quality of Experience (QoE) within the desired level. The remaining sections of this paper are organized as follows. Section II discusses the related work, followed by the methodology presentation in Section III. The experimental validation of the proposed methodology is performed in Section IV. Finally, Section V presents the conclusions and future work to be done.

\section{RELATED WORK}

This section presents the most relevant related work on energy-efficient video transmission and video end-user perceived quality. Li et al. [3] have performed a study of energyeficient video transmission over a wireless link, by controlling the parameters associated with the physical and link layers. The results showed energy savings of around 38\% for a CDMA system supporting six users. However, the assessment was fully based on simulations, which does not accurately represent real systems. Other simulation based works have proposed energy-efficiency approaches for video transmission based on scalable video coding features, using content-aware rate control techniques [4] and cooperative video transmission with end-to-end statistical Quality of Service provision [5]. Yuan el al. [6] have employed cross-layer techniques to improve multimedia application quality, while minimizing battery energy consumption. The proposed cross-layer solution was validated in a testbed using a digital oscilloscope for the energy related assessment, but it has not studied the impact of the approach in the end-user perceived quality. The evolution of video quality metrics has shown the importance of an accurate assessment of end-user QoE [7]. Many works have studied the impact of transmitting video within different network conditions in the QoE perceived by the end-user, but without any focus on energy consumption [8] [9]. In a previous work, Bernardo et al. [10] have assessed the energy consumption of generic cloud computing applications in an 
empirical testbed, by analyzing the impact of packet rate and packet size in the end-user system. However, the methodology presented does not have the capability to measure video energy consumption, neither the quality of experience perceived by the end-user.

To the best of our knowledge, there is not any integrated empirical methodology that is able to assess video quality while measuring energy consumption. By analyzing the stateof-the-art work on energy-efficient video transmission, there is a clear lack of an experimental evaluation methodology to assess and validate the proposed optimization mechanisms and algorithms. Besides validating, the experimental evaluation of video energy consumption and quality can also be an important contribution to design realistic simulation models.

\section{Methodology}

This section describes the methodology for assessing the video energy consumption and quality.

\section{A. Objectives}

The methodology aims to provide a framework that addresses, in a combined way, the energy consumption and the end-user perception of the video traffic, while satisfying the following requirements:

- High-precision energy consumption measurement of each transmitted video: to accurately study video streaming energy consumption, it is vital to use a hardware that is capable to support multiple samples per second, since energy in small devices (i.e. network interfaces) tends to have slight variations over time;

- Technology independent evaluation: the methodology must be technology independent, to enable the comparison and study of different wireless technologies;

- Evaluation under variable network conditions: to study different network conditions, namely by inserting random packet losses or limiting the available bandwidth;

- Quality of Experience assessment: the methodology must encompass metrics to perform end-user perceived quality evaluation, and to establish correlation with energy consumption values.

\section{B. Description}

The proposed methodology has three main components: energy measurement, video evaluation, and link emulator, as depicted in Figure 1.

1) Energy measurement: The employed energy measurement facility was designed to minimize the hardware changes in the system. The usage of an external USB network interface enables the possibility to use the system with various network interface types. The measurement configuration includes the end-user device, a "controller machine" and a high-precision digital multimeter. The digital multimeter is a Rigol DM3061 with a maximum sampling rate of $50 \mathrm{~K}$ samples/second and a test resolution of $61 / 2$ digits. The multimeter is capable of receiving Standard Commands for Programmable Instruments (SCPI) commands (defined by IEEE 488.2) and implements the Universal Serial Bus Test and Measurement Class

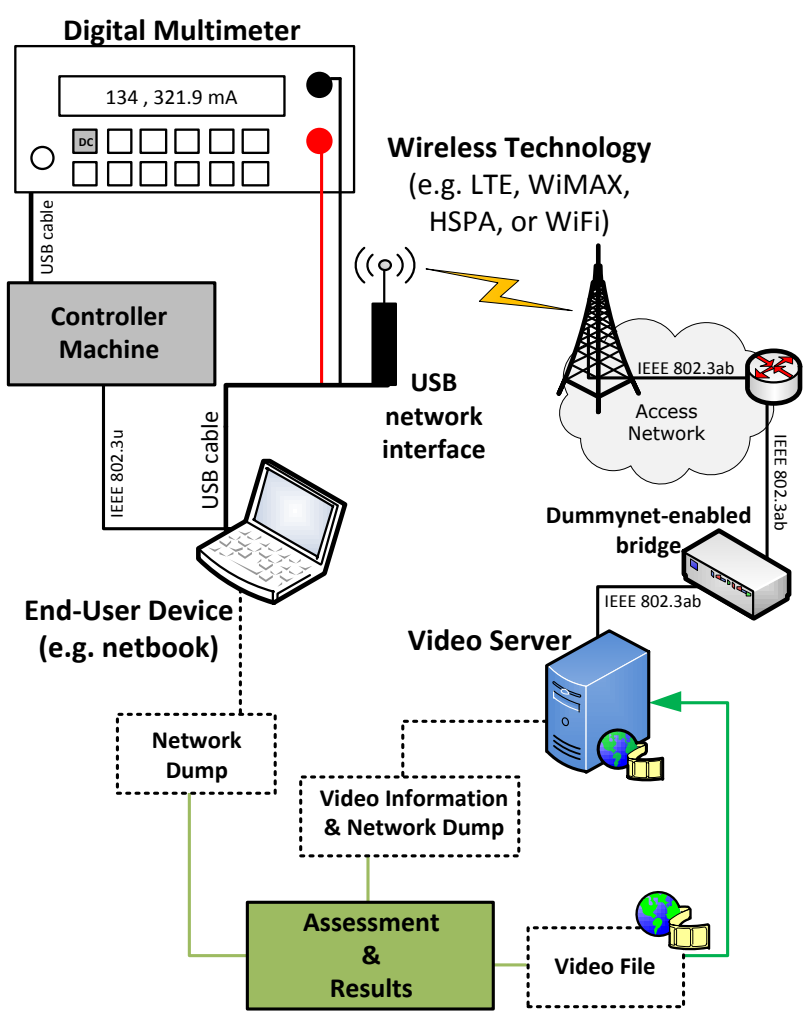

Fig. 1. Methodology architecture

Specification (USBTMC) standard interface. By using SCPI commands and USBTMC the "controller machine" is able to control and manage the digital multimeter, which enables accurate and repeatable test sets. The "controller machine" is also connected to the end-user device. This entity enables a fast and reliable point to control the experiments to be performed and to collect all the results from the digital multimeter. Since the voltage is stable, all the measurements concerning energy were done by collecting the current values. The USB cable was intercepted in the common-collector voltage (VCC) cable (i.e., +5 VDC).

2) Video traffic generation: Video traffic generation was done with the Evalvid framework [11], since this framework has the ability to reconstruct the received video, even when some frames have been lost. Moreover, Evalvid reports QoS metrics, such as packet loss and one-way delay, and QoE metrics, such as Mean Opinion Score (MOS). The integration of Evalvid within the proposed methodology is illustrated in Figure 1. The "Video Server" entity is responsible for the startup of the video streaming to the "end-user device" through the network. First, the video preparation is performed; a raw lossless YUV video is compressed to be sent to the enduser. Then, the sender is able to start the streaming, and simultaneously, it will begin to capture information about the transmitted video. Details such as frame types and sending times are collected with the Evalvid sending tool, and the wellknown tcpdump tool collects the network information about the transmitted packets. The same network capture is repeated 
in the receiver side until the video transmission ends.

When the video and the network information have been completely collected in both the sender and the receiver, the video is reconstructed at the receiver by using the collected information and the original transmitted video file. Then, the reconstructed coded video is transformed back into raw YUV format, in order to perform the video quality comparison with the original lossless raw YUV video.

3) Link emulator: The link emulator, implemented using a Dummynet-enabled bridge [12], provides a fully configurable environment, enabling the study of several network conditions. Therefore, the proposed methodology allows the emulation of controlled packet loss, delay or bandwidth values, where the environment is stable, and the tests can be repeated with the same conditions. This solution is completely transparent to all the equipment, since it acts as transparent bridge between the "Video Server" and its connection to the access network.

\section{Evaluation metrics}

The proposed methodology reports parameters about video energy consumption and quality. Energy is assessed through both power and energy consumption for a certain video transmission. Moreover, power can be analyzed as a function of time, to correlate video characteristics and power consumption. The energy consumption for a desired period (different from the total video playing time) can also be obtained. Video quality is assessed through packet or frame loss, delay, rate information and Peak Signal Noise to Ratio (PSNR). Additionally, video quality is also assessed through QoE metrics, such as MOS and Structural Similarity Index (SSIM).

MOS is a sensorial metric, which requires humans to evaluate the quality of the voice in a scale from 1 (bad quality) to 5 (excellent quality). This score is calculated based on the perceived quality of a certain number of users, and results are presented as average quality. SSIM [13] is an objective and full reference image quality metric, which measures the similarity between two images. It is based on three different similarity components, namely the contrast, the luminance and the structural similarity. Unlike PSNR, which is incoherent with Human Visual System characteristics, such as human eye perception, SSIM takes into consideration human eye perception parameters, which improves the evaluation accuracy. Therefore, the resulting SSIM Index is a combination of the three similarity parameters into a single value between 0 and 1 , where 0 means no correlation with the original image, and 1 means the exact same image.

The proposed methodology does not limit the usage of Evalvid for Quality of Experience assessment. Since the generated raw video file is available, the video comparison can be performed with any available tool.

\section{VALIDATION}

This section describes the experimental validation of the methodology performed in two distinct testbeds. The first subsection describes the video sequences used, followed by the experimental testbed overview. Later, the experimental validation results are discussed.

\section{A. Objectives}

The main goal of this experimental validation is to assess the capability of the proposed methodology to achieve accurate results in the empirical measurement of video energy consumption and end-user perceived quality, by analyzing the resultant Quality of Experience. To fulfill these requirements six compressed different formats of a high quality lossless video were created. The empirical evaluation was performed in two real testbeds, encompassing two different wireless access technologies and three distinct access network condition scenarios.

\section{B. Video sequences}

The video sequences used were extracted from the TUM Multi Format Test Set [14], which consists of 48 different video sequences in multiple formats, including HDTV. The "carving4" video was selected to perform this evaluation. This sequence has a resolution of $1920 \times 1080$ pixels, a frame rate of 25 frames per seconds, and contains 300 frames. A focus modification within a shot with several carved figures is shown during the 12 seconds of duration.

The video used in this evaluation was compressed with the MPEG-4 codec. This codec uses three different types of frames, namely, I-frames, P-frames and B-frames. All the video related operations were performed using ffmpeg software [15].

The "carving4" sequence used in this assessment was compressed in different ways, including five Constant Bit Rate (CBR) videos and one Variable Bit Rate (VBR), which aims to provide the same quality as the original sequence. Additionally, all the movies were encoded using a GOP of 30 frames with 25 Frames Per Second (FPS). Table I shows the most relevant parameters of each compressed video.

TABLE I

COMPRESSED VIDEO SEQUENCES

\begin{tabular}{lcccc} 
Name & Type & Bitrate & PSNR & SSIM \\
\hline \hline carving4-CBR-2Mbps & CBR & $2155 \mathrm{~kb} / \mathrm{s}$ & 42.69 & 0.97 \\
carving4-CBR-4Mbps & CBR & $4181 \mathrm{~kb} / \mathrm{s}$ & 44.20 & 0.98 \\
carving4-CBR-6Mbps & CBR & $6062 \mathrm{~kb} / \mathrm{s}$ & 44.76 & 0.98 \\
carving4-CBR-8Mbps & CBR & $8054 \mathrm{~kb} / \mathrm{s}$ & 45.16 & 0.98 \\
carving4-CBR-10Mbps & CBR & $10053 \mathrm{~kb} / \mathrm{s}$ & 45.52 & 0.98 \\
carving4-VBR & VBR & Peak Rate: & 46.00 & 0.98 \\
& & $12956 \mathrm{~kb} / \mathrm{s}$ & &
\end{tabular}

\section{Experimental testbed}

This subsection presents the University of Coimbra IEEE 802.16e (Mobile WiMAX) and IEEE 802.11 (WiFi) testbeds, illustrated in Figure 2. The IEEE 802.11 access network is composed by a IEEE 802.11n router (Cisco Linksys E4200) and USB network interface (Cisco Linksys AE1000), both operating in the $2.4 \mathrm{GHz}$ band. The Mobile WiMAX testbed is a real deployment located in the city of Coimbra, Portugal, 
encompassing a Base Station (BS) and various Customer Premises Equipment (CPE). The Mobile WiMAX BS is an Alvarion BreezeMAX Macro Outdoor Network Access Unit with two $65^{\circ}$ sector dual polarization antennas with maximum transmission (TX) power of $38 \mathrm{dBm}$, configured to operate in the $2610.00 \mathrm{MHz}$ central frequency. The CPE used in the tests was an Alvarion USB BreezMAX 250. Moreover, the IEEE 802.16e testbed is fully compliant with the WiMAX Forum Network Reference Model [16]. In order to measure

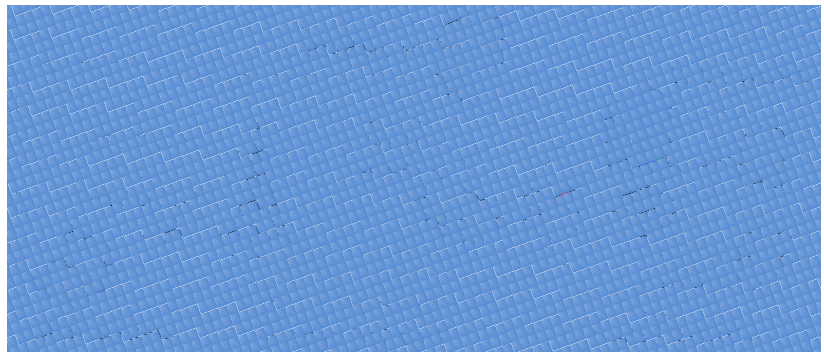

Fig. 2. IEEE 802.16e (Mobile WiMAX) and IEEE 802.11 (WiFi) testbeds

one-way end-to-end delay, system clocks of the end-hosts were synchronized with an open-source implementation of the IEEE 1588 Precision Time Protocol (PTP), the PTPd [17]. PTPd provides synchronization accuracy in the magnitude of submilliseconds, with coordination in the order of $10 \mu \mathrm{s}$ [18]. All the hosts use a dedicated network card for the exchange of PTP messages, to guarantee that the synchronization traffic does not introduce overhead on the wireless link.

An Asus EEE 1001PX-H netbook was used as "Mobile Node" equipment, running Ubuntu Linux kernel version 2.6.32-21generic. The "Video Server" machine, located in the core network, is a HP ProLiant DL320 G5p server running Debian Linux kernel version 2.6.32-5-amd64. The Dummynet-enabled bridge runs over FreeBSD 8.0.

The traffic referred as "receiving" $(\mathrm{RX})$ is generated by the "Video Server" machine in the core network and received by the "Mobile Node" in each scenario. The "transmitting" (TX) term is used to express the traffic with source on "Mobile Node" and with "Video Server" as destination. All results presented in the following sections are measured according to the proposed methodology, and include 10 runs for each test setup with a confidence interval of $95 \%$. The energy consumption was calculated by measuring the power consumption using a rate of $50 \mathrm{~K}$ samples.

\section{Experimental validation results}

This subsection presents the results from the experimental validation performed in the testbed.

The maximum throughput than can be attained in both WiFi and WiMAX testbeds was measured. The maximum downlink and uplink throughput for WiFi were 91.6 Mb/s and $87.3 \mathrm{Mb} / \mathrm{s}$, respectively. In the WiMAX scenario, the maximum throughput for downlink and uplink were $4.7 \mathrm{Mb} / \mathrm{s}$ and $3.8 \mathrm{Mb} / \mathrm{s}$, respectively. The results obtained with WiMAX were due to the non line of sight conditions of the deployed network.
1) WiFi native scenario: In this scenario the WiFi testbed was used, and the Dummynet bridge was disabled. Therefore, the tests were done using the best possible network conditions. Figure 3 depicts the total energy consumed for transmitting / receiving each compressed "carving4" video sequence (i.e., 12 seconds) in Joule. The $\mathrm{x}$-axis represents the video bitrate, according to the information presented in Table I. The energy needed to transmit a video is slightly higher than to receive the same video, and increases with the bitrate.

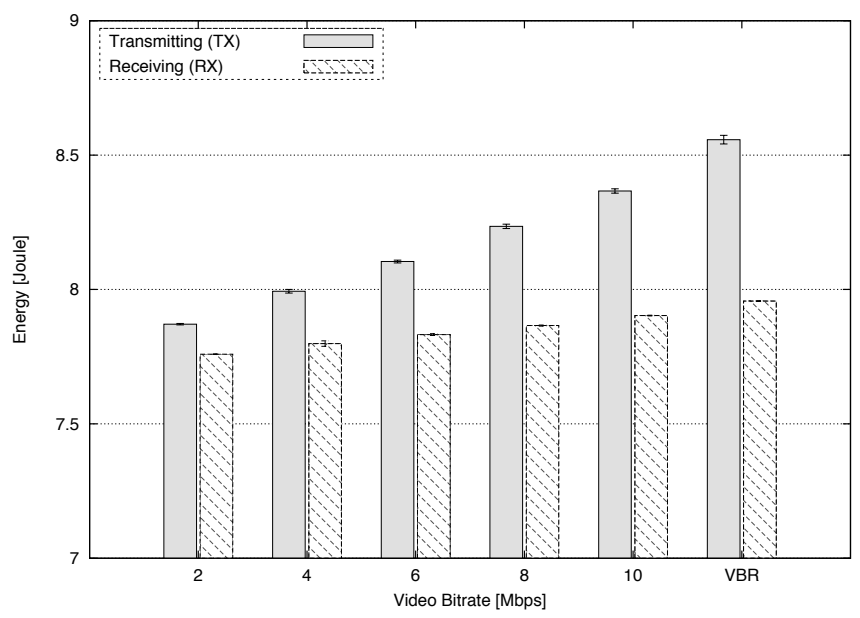

Fig. 3. Energy consumption of "carving4" video sequences over WiFi

The end-user perceived quality metric, expressed by the SSIM metric, is illustrated in Figure 4. The SSIM of all videos is

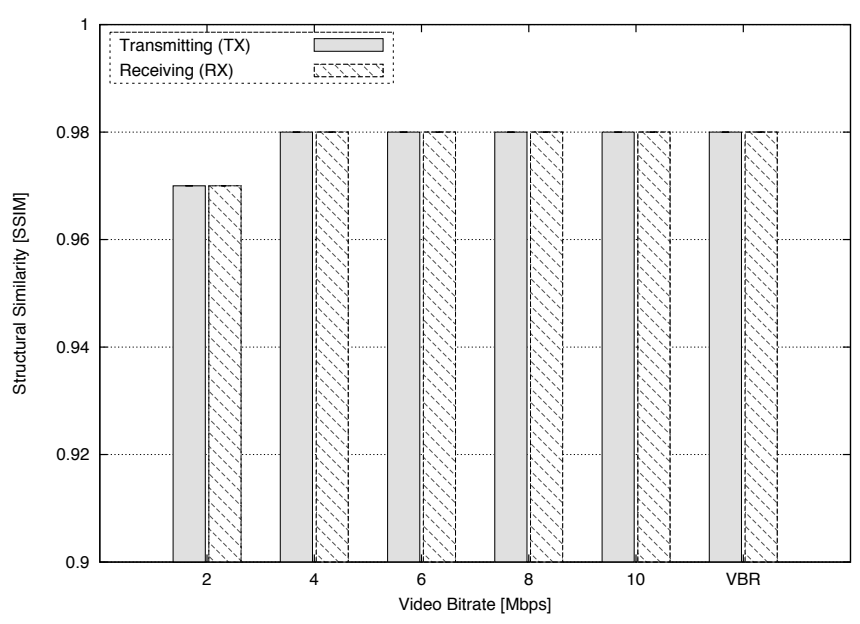

Fig. 4. SSIM of "carving4" video sequences over WiFi

roughly the same as each correspondent reference video. Even with very good network conditions, as in this scenario, with low one-way delay and without losses, the SSIM does not achieve the maximum value (i.e., $\mathrm{SSIM=1)}$ ). The maximum SSIM value for each compressed video is shown in Table I. These values are limited by the quality obtained by the configured compression rate. The impact of the video bitrate on the energy consumption is clear. The energy savings for transmitting the "carving4-CBR-2Mbps" video when compared with 
the "carving-CBR-10Mbps" is around 5.86\%. However, due to the "carving4" video characteristics, the quality perceived by the end-user when playing both videos is almost the same, since the SSIM is 0.97 and 0.98 , respectively.

2) WiMAX scenario: This scenario uses the already presented WiMAX testbed, without any limitation in Dummynetenabled bridge. Figure 5 shows the energy consumption of "carving4" sequences for the WiMAX scenario.

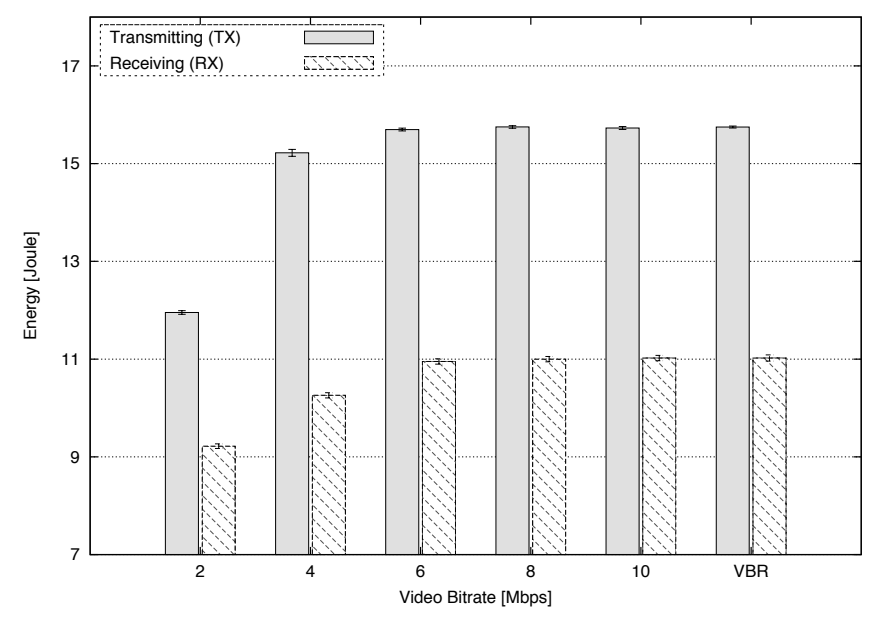

Fig. 5. Energy consumption of "carving4" video sequences over WiMAX

When compared with WiFi, WiMAX uses more energy for transmitting / receiving the same sequence. The gap between transmitting and receiving a video is also more noticeable in the WiMAX network. However, when "carving4" sequences with a rate equal or higher than $6 \mathrm{Mb} / \mathrm{s}$ are being transmitted or received, the energy consumption is roughly the same. This behavior can be explained by the increased amount of video frame losses. When the WiMAX maximum throughput is reached, the network interface will not be able to receive (or send) all the desired data and, as a result, it will not need additional energy. Figure 6 shows a detailed analysis

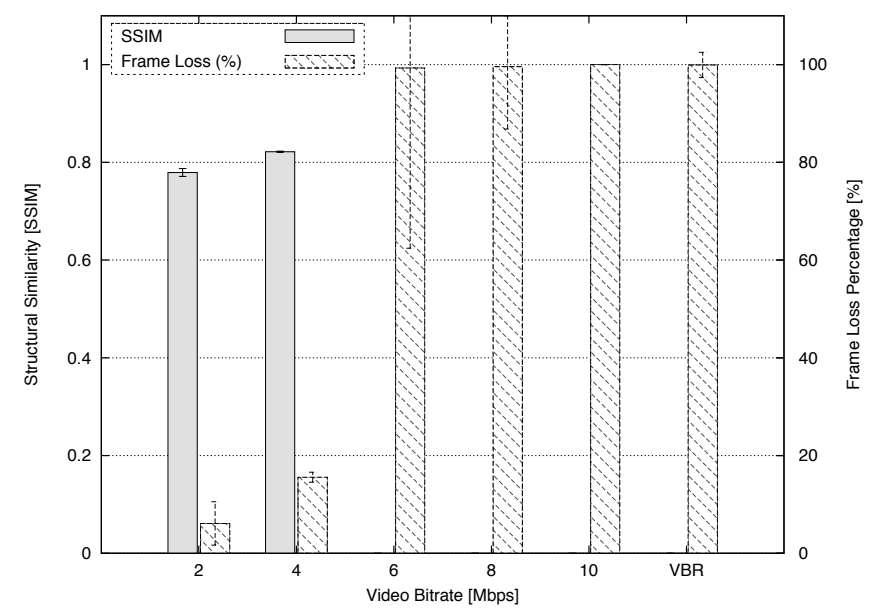

Fig. 6. SSIM and Frame Loss Rate when receiving (RX) "carving4" sequences over WiMAX of frame loss rate (right y-axis) when receiving (RX) "carving4" sequences over WiMAX. The end-user perception of the received video is given by the SSIM, depicted in the left y-axis. By analyzing the frame losses, it is clear that WiMAX cannot transport a video with bitrate around $6 \mathrm{Mb} / \mathrm{s}$. All the sequences with bitrate equal or higher than $6 \mathrm{Mb} / \mathrm{s}$ result on a SSIM value of zero, because it was not possible to reconstruct the received videos with such frame loss rate. These results show the importance of accurate mechanisms to control the video quality and energy consumption, since higher rate videos do not bring any advantage, neither concerning energy consumption nor end-user perceived quality.

3) Link Emulator - WiFi with similar WiMAX bandwidth and delay: This scenario uses the WiFi testbed with the Dummynet-enabled bridge configured to limit the available bandwidth in order to have a throughput similar to the WiMAX testbed. Since one-way delay in WiMAX is also higher than in WiFi, the link emulator configuration introduces the additional delay needed to reproduce WiMAX conditions. Moreover, a random packet loss rate of $0.05 \%$ was applied to emulate the packet loss rate in WiMAX, due to the deployment in non line of sight conditions and to the resultant bit rate error. The configuration was tested with iperf and ping Linux tools.

Figure 7 depicts the energy consumption of receiving (RX) "carving4" sequences over WiFi using the link emulator configured to limit the WiFi bandwidth and increase the oneway delay. The results show a clear difference on the energy

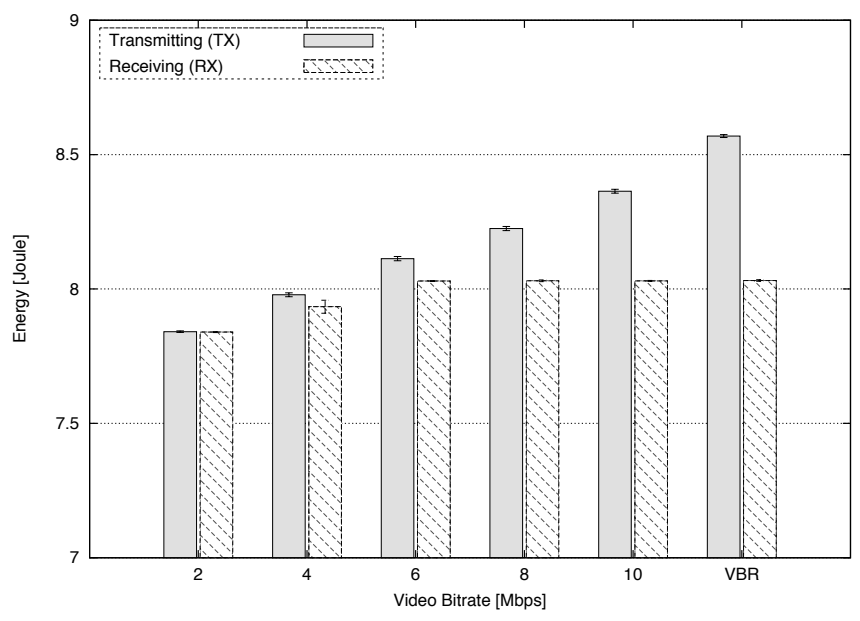

Fig. 7. Link Emulator: Energy consumption of "carving4" sequences over WiFi with same bandwidth and delay as WiMAX

consumption. As expected, since the WiFi USB network interface was the same, the energy consumed by WiFi is similar to the native WiFi scenario (see Figure 3). The frame loss percentage and the SSIM for this scenario are illustrated in Figure 8. The frame loss percentage is roughly the same as in the WiMAX scenario. Again, the SSIM for video with higher frame loss is zero. In the case of "carving4-CBR-4Mbps", the frame loss in WiFi with active link emulator is lower, but the SSIM is also lower. This can be explained by the type of frames lost, since I-frames (i.e., key frames) will have a higher 


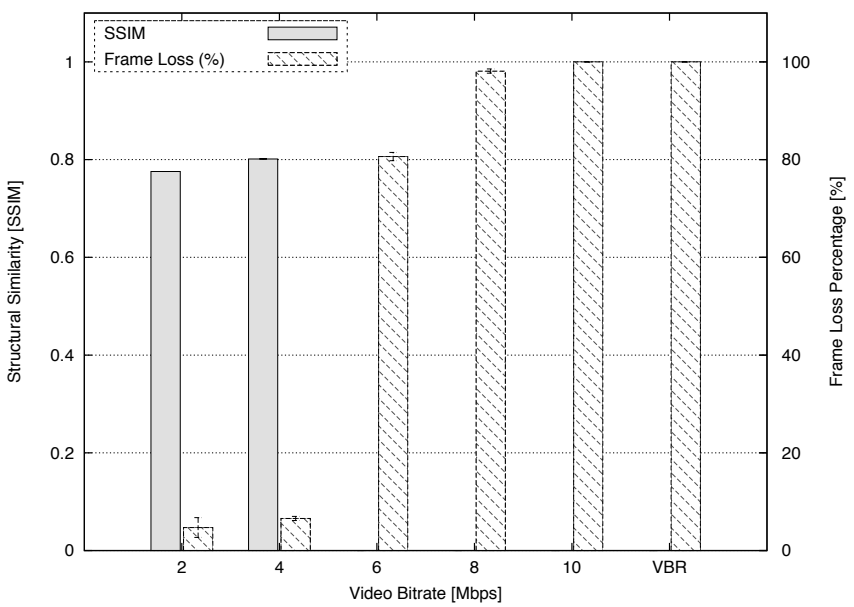

Fig. 8. Link Emulator: SSIM and Frame Loss Rate when receiving (RX) "carving4" sequences over WiFi with same bandwidth and delay as WiMAX

impact in the end-user perceived quality than, for instance, $\mathrm{P}$ frames.

\section{CONCLUSION}

This paper has proposed an integrated empirical methodology to assess energy consumption and video quality within heterogeneous networks. The proposed solution can be used with any USB network device, creating novel opportunities to compare and study both legacy and state-of-the-art wireless access network technologies. By using the proposed methodology, an experimental testbed validation encompassing two distinct wireless access networks was performed. The importance of the integrated measurement of energy consumption and video quality, as perceived by the end-user, was supported by the results obtained. Moreover, the methodology has shown the capability to create heterogeneous network conditions, being able to emulate configured bandwidth, delay or packet loss values. Regarding the studied technologies, the results showed that WiMAX is more energy demanding than WiFi for the studied scenarios. The results also showed that, even without implementing any optimization mechanism, it is possible to reduce energy consumption without affecting the end-user Quality of Experience, by just adapting the video bitrate to the network conditions.

In future work, the developed methodology will be used to assess novel video transmission energy optimization techniques, taking into account the end-user Quality of Experience. Additionally, the empirical data obtained using this methodology can also be used to create or improve simulation models.

\section{ACKNOWLEDGMENT}

Special thanks to Prof. Dr. Torsten Braun for all the support during this work.

This work was partially supported by the Portuguese National Foundation for Science and Technology (FCT) through a Doctoral Grant (SFRH/BD/66181/2009) and by a Short-Term Scientific Mission from the COST Action IC0906 "Wireless
Networking for Moving Objects (WiNeMO)". Thanks also to EDPWiMAX project, funded by EDP Distribuição S.A.

\section{REFERENCES}

[1] M. Zuhdi, E. Pereira, and A. Teixeira, "Trends in the telecom industry and opportunities for service providers," in Transparent Optical Networks (ICTON), 2011 13th International Conference on, june 2011, pp. $1-4$.

[2] R. Bolla, R. Bruschi, F. Davoli, and F. Cucchietti, "Energy efficiency in the future internet: A survey of existing approaches and trends in energy-aware fixed network infrastructures," Communications Surveys Tutorials, IEEE, vol. 13, no. 2, pp. 223 -244, quarter 2011.

[3] Y. Li, M. Reisslein, and C. Chakrabarti, "Energy-efficient video transmission over a wireless link," Vehicular Technology, IEEE Transactions on, vol. 58, no. 3, pp. 1229-1244, march 2009.

[4] S. Lee, J. Koo, and K. Chung, "Content-aware rate control scheme to improve the energy efficiency for mobile iptv," in Consumer Electronics (ICCE), 2010 Digest of Technical Papers International Conference on, jan. 2010, pp. $445-446$.

[5] A. Abdel Khalek and Z. Dawy, "Energy-efficient cooperative video distribution with statistical qos provisions over wireless networks," Mobile Computing, IEEE Transactions on, vol. PP, no. 99, p. 1, 2011.

[6] W. Yuan, K. Nahrstedt, S. Adve, D. Jones, and R. Kravets, "Grace1: cross-layer adaptation for multimedia quality and battery energy," Mobile Computing, IEEE Transactions on, vol. 5, no. 7, pp. $799-815$, july 2006.

[7] S. Winkler and P. Mohandas, "The evolution of video quality measurement: From psnr to hybrid metrics," Broadcasting, IEEE Transactions on, vol. 54, no. 3, pp. $660-668$, sept. 2008.

[8] K. De Moor, I. Ketyko, W. Joseph, T. Deryckere, L. De Marez, L. Martens, and G. Verleye, "Proposed framework for evaluating quality of experience in a mobile, testbed-oriented living lab setting," Mobile Networks and Applications, vol. 15, pp. 378-391, 2010, 10.1007/s11036010-0223-0. [Online]. Available: http://dx.doi.org/10.1007/s11036-0100223-0

[9] V. Bernardo, K. Pentikousis, J. Pinola, E. Piri, and M. Curado, "Multi-client video streaming over wirelessman-ofdma," in Proceedings of the 4th ACM workshop on Performance monitoring and measurement of heterogeneous wireless and wired networks, ser. PM2HW2N '09. New York, NY, USA: ACM, 2009, pp. 46-53. [Online]. Available: http://doi.acm.org/10.1145/1641913.1641920

[10] V. Bernardo, M. Curado, T. Staub, and T. Braun, "Towards energy consumption measurement in a cloud computing wireless testbed," in Network Cloud Computing and Applications (NCCA), 2011 First International Symposium on, nov. 2011, pp. 91 -98. [Online]. Available: http://dx.doi.org/10.1109/NCCA.2011.22

[11] J. Klaue, B. Rathke, and A. Wolisz, "EvalVid - A Framework for Video Transmission and Quality Evaluation," in Proceedings of the 13th Conference on Modelling Techniques and Tools for Computer Performance Evaluation, ser. Lecture Notes in Computer Science. Springer, 2003, pp. 255-272. [Online]. Available: http://www.tkn.tu-berlin.de/publications/papers/evalvid.pdf AND http://citeseer.ist.psu.edu/klaue03evalvid.html

[12] M. Carbone and L. Rizzo, "Dummynet revisited," SIGCOMM Comput. Commun. Rev., vol. 40, pp. 12-20, April 2010. [Online]. Available: http://doi.acm.org/10.1145/1764873.1764876

[13] Z. Wang, A. C. Bovik, and H. R. Sheikh, "Image quality assessment: From error measurement to structural similarity," IEEE Trans. Image Processing, vol. 13, January 2004.

[14] Technische Universität München, Institute for Data Processing. (2011) TUM LDV Multi Format Test Set. [Online]. Available: http://www.ldv.ei.tum.de/videolab

[15] S. Tomar, "Converting video formats with ffmpeg," Linux J., vol. 2006, pp. 10-, Jun. 2006. [Online]. Available: http://dl.acm.org/citation.cfm?id=1134782.1134792

[16] K. Etemad, "Overview of mobile wimax technology and evolution," Communications Magazine, IEEE, vol. 46, no. 10, pp. $31-40$, oct 2008 .

[17] K. Correl. (2012) Ptp daemon (ptpd). [Online]. Available: http://ptpd.sourceforge.net. Last visit: 2 January 2012

[18] K. Correll and N. Barendt, "Design considerations for software only implementations of the ieee 1588 precision time protocol," in In Conference on IEEE 1588 Standard for a Precision Clock Synchronization Protocol for Networked Measurement and Control Systems, 2006. 